\title{
Association of the FRAIL Scale with Geriatric Syndromes and Health- Related Outcomes in Korean Older Adults
}

\author{
Heayon Lee ${ }^{1,2, *}$, Junyong Chong ${ }^{3, *}$, Hee-Won Jung ${ }^{1}$, Ji Yeon Baek ${ }^{1}$, Eunju Lee ${ }^{1}$, Il-Young Jang ${ }^{1,4}$ \\ ${ }^{1}$ Division of Geriatrics, Department of Internal Medicine, Asan Medical Center, University of Ulsan College of Medicine, Seoul, Korea \\ ${ }^{2}$ Division of Pulmonary, Critical Care and Sleep Medicine, Department of Internal Medicine, Eunpyeong St. Mary's Hospital, College of Medicine, The Catholic \\ University of Korea, Seoul, Korea \\ ${ }^{3}$ Division of Gastroenterology, Department of Internal Medicine, Asan Medical Center, University of Ulsan College of Medicine, Seoul, Korea \\ ${ }^{4}$ Pyeongchang Health Center \& Country Hospital, Gangwon-do, Korea
}

Corresponding Author:

Il-Young Jang, MD

Department of Internal Medicine, Asan

Medical Center, University of Ulsan

College of Medicine, 88 Olympic-ro

43-gil, Songpa-gu, Seoul 05505, Korea

E-mail: onezero2@gmail.com

ORCID:

https://orcid.org/0000-0003-3617-3301

Received: December 11,2020

Revised: April 20, 2021

Accepted: May 5, 2021

${ }^{*}$ These authors contributed equally to this work.
Background: Owing to the growing older population, appropriate tools are needed for frailty screening in community-dwelling older people. We investigated the association between geriatric conditions and health-related outcomes using the five-item Fatigue, Resistance, Ambulation, Illnesses, \& Loss of Weight (FRAIL) scale in a Korean rural community setting. Methods: We performed comprehensive geriatric assessments, including the FRAIL scale, in 1,292 community-dwelling people (mean age, 74.6 years) in the Aging Study of Pyeongchang Rural Area. These populations were prospectively followed up for 3 years to analyze the outcomes of death, institutionalization, disability, and quality of life. We investigated the association between frailty status and outcomes using the FRAIL scale. Results: According to the FRAIL scale, 524 (36.5\%) participants were prefrail and 297 (23.0\%) were frail. According to the adjusted model, the degree of frailty status was significantly associated with concurrent geriatric syndromes and 3-year incidences of mortality, institutionalization, and disability; Kaplan-Meier analysis showed significant differences in 3-year survival based on frailty status (92.6\% for robust, $85.7 \%$ for prefrail, and $74.2 \%$ for frail; log-rank $p<0.001$ ). Conclusion: The five-item FRAIL scale can be used to screen for accompanying geriatric syndromes and is associated with the 3-year health-related outcomes in community-dwelling Korean older adults. From the public health perspective, this simple screening tool for frailty assessment might be applicable to older populations in Korea.

Key Words: Frailty, Outcome assessment, Health care, Public health, Mass screening

\section{INTRODUCTION}

South Korea is one of the fastest aging societies worldwide; moreover, the estimated life expectancy of Korean people is the highest (in 2030) among people from developed countries. ${ }^{11}$ However, according to the 2018 Korean Geriatrics Fact Sheet, $89.5 \%$ of older Korean adults had one or more chronic diseases and the annual national medical expenses for older adults accounted for 39.9\% of the total medical expenses. ${ }^{2)}$ With these changes in population, there is an overarching need to identify vulnerable older adults at high risk for conditions such as multimorbidity, polypharmacy, low economic status, cognitive impairment, and functional decline; moreover, growing evidence suggests that frailty is a core geriatric syndrome that is highly correlated with human aging, multimorbidity, and disability. ${ }^{3-7)}$ Frailty is defined as a state of decreased physiological reserve associated with human aging and is a strong predictor of adverse health outcomes such as falls, fractures, loss of independence, disability, institutionalization, and death. ${ }^{8,9)}$ Therefore, screening for frailty in older populations is a crucial step in delivering tailored multifaceted care for highly heterogeneous 
geriatric care needs among individuals. ${ }^{10}$

Currently, the most commonly used frailty definitions in research $^{3,11}$ are the frailty phenotype and frailty index. These tools are less feasible for use in mass populations as they are resource intensive, research oriented, and especially troublesome to adapt to policies of resource-limited community settings. ${ }^{12)}$ As a screening measure, the Fatigue, Resistance, Ambulation, Illnesses, and Loss of weight (FRAIL) scale, a self-reported screening instrument for frailty developed by Morley et al., ${ }^{13)}$ is a valid method for use in communities. This short questionnaire is easy to administer, requires minimal time and resources, and has comparable performance in screening frailty. It has been validated in various populations and is a useful screening tool for identifying frail older adults. ${ }^{13-15)}$ The Korean version, the K-FRAIL scale, has been validated in the Korean population, especially in older adults in urban communities. ${ }^{16,17)}$ The FRAIL scale has been shown to have predictive validity for mortality in various studies. ${ }^{18,19)}$ However, other health outcomes such as disability or institutionalization require further investigation. ${ }^{20)}$

Thus, this study examined the association between geriatric conditions and health-related outcomes such as mortality, institutionalization, and disability using the five-item FRAIL scale in rural community-dwelling Korean older adults.

\section{MATERIALS AND METHODS}

\section{Study Population}

We analyzed the records of participants in the Aging Study of Pyeongchang Rural Area (ASPRA) cohort, a population-based, prospective cohort study originally established in 2014 that included community-dwelling adult residents of Pyeongchang-gun, Gangwon Province, South Korea who were aged 65 years and older. Detailed information on the original study design and measurements in the ASPRA has been published elsewhere. ${ }^{21)}$ During annual assessments, the study population continuously increased with the expansion of the covered region of ASPRA among Pyeongchang-gun, with the inclusion and exclusion criteria remaining the same. Individuals were eligible for participation if they were (1) aged 65 years or older, (2) registered in the Community Healthcare Service, (3) ambulatory with or without an assistive device, (4) living at home, and (5) able to provide informed consent by themselves or their legal proxies. We excluded participants who were (1) living in nursing homes, (2) hospitalized, or (3) bedridden and receiving nursing home level care at home at the time of enrollment.

Between December 2014 and December 2017, 1,295 community-dwelling individuals were enrolled in the ASPRA cohort, which included over $90 \%$ of eligible people living in the study area. Among these older adults, we used records of 1,292 people who completed the K-FRAIL questionnaire. All participants underwent a comprehensive geriatric assessment every 12 months, while health-related outcomes, including death, institutionalization, and disability, were recorded every 3 months. The characteristics of the original ASPRA population were generally comparable to those of the general Korean rural population based on the Korea National Health and Nutrition Examination Survey. ${ }^{21)}$ The study protocol of the ASPRA cohort was approved by the Institutional Review Board of the Asan Medical Center (No. 2015-0673). Written informed consent was obtained from all participants.

\section{Frailty Screening}

We used the K-FRAIL questionnaire as a screening measure for frailty status. The K-FRAIL is based on the original FRAIL scale, ${ }^{13)}$ which has been validated in various populations and translated into Korean. ${ }^{16)}$ This simple screening tool of five self-reported items can be assessed in 2-3 minutes with no requirement for special equipment or sophisticated training. The questionnaire comprises five items:

(1) Fatigue (responses of "all of the time" or "most of the time" to the following question): "How much of the time during the past 4 weeks did you feel tired?"

(2) Resistance (positive response to the question): "By yourself and not using aids, do you have any difficulty in walking up 10 steps without resting?"

(3) Ambulation (positive response to the question): "By yourself and not using aids, do you have any difficulty in walking $300 \mathrm{~m}$ ?"

(4) Illnesses (five or more self-reported physician diagnoses of hypertension, diabetes, cancer excluding minor skin cancer, chronic lung disease, heart attack, congestive heart failure, angina, asthma, arthritis, stroke, or kidney disease), and

(5) Loss of weight ( $>5 \%$ in the past year).

We calculated the K-FRAIL score by combining the number of positive items and classified the participants as robust ( 0 points), prefrail (1-2 points), or frail (3-5 points).

\section{Assessment of Geriatric Conditions}

Trained nurses performed comprehensive geriatric assessments (CGAs) to evaluate geriatric conditions. Multimorbidity was defined as having two or more of the 11 physician-diagnosed illnesses such as angina, arthritis, asthma, cancer, chronic lung disease, congestive heart failure, diabetes, hypertension, heart attack, kid- 
ney disease, and stroke. Polypharmacy was defined as regularly taking five or more medications. ${ }^{22)}$ Cognitive impairment was defined as a Korean version of the Mini-Mental State Examination score of $<24 .{ }^{23)}$ Depressive mood was defined as a Korean version of the Center for Epidemiological Studies Depression scale score of $>20{ }^{24)}$ Malnutrition risk was defined as a Mini-Nutritional Assessment-Short Form score of $\leq 11$. $^{25)}$ To assess physical function, we measured handgrip strength $(\mathrm{kg})$ using a dynamometer (T.K.K. 5401 Grip-D; Takei, Tokyo, Japan) and averaged two measurements of the dominant hand. ${ }^{21)}$ We defined dysmobility as a slow gait (usual gait speed $<0.6 \mathrm{~m} / \mathrm{s}$ from a timed $4-\mathrm{m}$ walk). ${ }^{26)}$ We calculated the Short Physical Performance Battery (SPPB) score from three components: the ability to stand for up to $10 \mathrm{sec}-$ onds with feet positioned in three ways (together side-by-side, semi-tandem, and tandem), the time to complete a 4-m walk, and the time to rise from a chair five times. The resultant SPPB scores ranged from 0 to 12, with higher scores indicating a higher level of function. ${ }^{27)}$ Sarcopenia was defined according to the Asian Working Group for Sarcopenia as follows: decreased muscle mass (decreased handgrip strength of $<26 \mathrm{~kg}$ for men and $<18 \mathrm{~kg}$ for women) and decreased physical performance (slow gait of $<0.8$ $\mathrm{m} / \mathrm{s}) .{ }^{28)}$ Disability was defined as requiring assistance in performing any of the seven activities of daily living (ADL; bathing, continence, dressing, eating, toileting, transferring, and washing face and hands) or 10 instrumental activities of daily living (IADL; food preparation, household chores, going out on a short distance, grooming, handling finances, laundry, managing own medications, shopping, transportation, and using a telephone). ${ }^{21)}$

\section{Outcome Measurements}

This study defined major adverse health outcomes as death, institutionalization, and incidence of disability during the 3-year follow-up period. We defined disability as the requirement for assistance from another person in performing any one of the $\mathrm{ADL}$ or IADL. ${ }^{21)}$ Health-related quality of life was assessed during the follow-up period using the EuroQol-5D (EQ-5D). ${ }^{29)}$ An EQ-5D score decline over 3 years of $>0.2$ was considered clinically meaningful. ${ }^{30)}$ To assess these outcome measures, all participants underwent structured interviews via phone calls every 3 months on institutionalization, death, and annualized follow-up assessments, including CGAs for ADL, IADL, and EQ-5D.

\section{Statistical Analysis}

To compare variables for baseline characteristics according to the frailty status derived from the K-FRAIL scale, we used KruskalWallis and chi-square tests for continuous and discrete variables, respectively. To cross-sectionally assess the association between prevalent frailty and geriatric syndrome, we used logistic regression analysis with common geriatric syndromes as dependent variables and frailty status (robust, prefrail, and frail) as independent variables after adjusting for age and sex. For longitudinal outcome analysis, we used logistic regression analysis for health outcomes of mortality, long-term institutionalization, incidence of disability, and decline in EQ-5D score at 3 years from baseline, with frailty status (robust, prefrail, and frail) as dependent variables after adjusting age and sex. Mortality was further analyzed using KaplanMeier analysis, with log-rank tests and Cox proportional hazard regression analysis used to examine the statistical differences in survival according to the baseline frailty status. The restricted mean survival time was estimated as death at 40 months. Two-sided p-values $<0.05$ indicated statistical significance in all analyses. Statistical analyses were performed using IBM SPSS Statistics for Windows, version 21.0 (IBM Corp., Armonk, NY, USA).

\section{RESULTS}

\section{Baseline Characteristics according to Frailty Status based on the FRAIL Scale}

As shown in Table 1, 471 (36.5\%) participants were classified as robust, $524(40.5 \%)$ as prefrail, and 297 (23\%) as frail according to the K-FRAIL scale. The frail group was older, more likely to be female, more likely to be living alone, and had lower income than the other two groups $(\mathrm{p}<0.001)$. The frail group also had a higher burden of multimorbidity and polypharmacy and was more likely to have depression and cognitive impairment than the other two groups $(\mathrm{p}<0.001)$. Physical performance was worse and the prevalence of disability was higher in the frail group than in the other two groups $(\mathrm{p}<0.001)$.

\section{Associations between Frailty Status and Geriatric Conditions}

In Table 2, frailty according to the FRAIL scale was associated with geriatric conditions after adjusting for age and sex. The frail group showed a significant association with multimorbidity (odds ratio $[\mathrm{OR}]=2.24$; 95\% confidence interval [CI], 1.57-3.21), dysmobility $(\mathrm{OR}=5.35 ; 95 \% \mathrm{CI}, 3.58-7.97)$, malnutrition $(\mathrm{OR}=4.09$; 95\% CI, 2.83-5.90), cognitive impairment ( $\mathrm{OR}=3.52$; 95\% CI, 2.36-5.25), depression ( $\mathrm{OR}=27.50 ; 95 \% \mathrm{CI}, 11.09-68.19)$, and disability (OR=3.12; 95\% CI, 2.18-4.46).

\section{Associations between FRAIL Scale and Health Outcomes}

Longitudinal analysis showed that baseline frailty status significantly increased the risks of mortality $(\mathrm{OR}=3.57$; 95\% CI, 1.69$7.54)$, institutionalization $(\mathrm{OR}=2.42 ; 95 \% \mathrm{CI}, 1.30-4.51)$, and disability at 3 years $(\mathrm{OR}=3.04 ; 95 \% \mathrm{CI}, 1.91-4.85)$ but not the 
Table 1. Baseline characteristics according to frailty status in the Korean version of the FRAIL scale

\begin{tabular}{|c|c|c|c|c|}
\hline & Robust $(n=471)$ & Prefrail $(n=524)$ & Frail $(n=297)$ & p-value ${ }^{a}$ \\
\hline Age (y) & $72.2 \pm 5.2$ & $74.6 \pm 6.1$ & $78.3 \pm 6.3$ & $<0.001$ \\
\hline Sex, female & $188(40.0)$ & $312(59.5)$ & $217(73.1)$ & $<0.001$ \\
\hline Living alone & $91(19.3)$ & $149(28.4)$ & $101(34.0)$ & $<0.001$ \\
\hline Low income & $21(4.5)$ & $37(7.1)$ & $30(10.1)$ & 0.010 \\
\hline $\operatorname{BMI}\left(\mathrm{kg} / \mathrm{m}^{2}\right)$ & $24.6 \pm 3.3$ & $24.5 \pm 3.7$ & $24.3 \pm 3.8$ & 0.534 \\
\hline Multimorbidity & $139(29.5)$ & $210(40.1)$ & $168(56.6)$ & $<0.001$ \\
\hline Polypharmacy & $73(15.5)$ & $118(22.5)$ & $107(36.0)$ & $<0.001$ \\
\hline Cognitive dysfunction & $62(13.2)$ & $164(31.3)$ & $157(52.9)$ & $<0.001$ \\
\hline Depressive mood & $6(1.3)$ & $49(9.4)$ & $87(29.3)$ & $<0.001$ \\
\hline At risk of malnutrition & $1(0.0)$ & $20(3.8)$ & $43(14.5)$ & $<0.001$ \\
\hline SPPB score & $9.7 \pm 2.3$ & $8.0 \pm 2.8$ & $5.0 \pm 3.1$ & $<0.001$ \\
\hline Dysmobility & $61(13.0)$ & $133(25.4)$ & $169(56.9)$ & $<0.001$ \\
\hline Sarcopenia & $78(16.6)$ & $185(35.3)$ & $145(48.8)$ & $<0.001$ \\
\hline ADL disability & $17(3.6)$ & $76(14.5)$ & $86(29.0)$ & $<0.001$ \\
\hline IADL disability & $111(23.6)$ & $149(28.4)$ & $176(59.3)$ & $<0.001$ \\
\hline
\end{tabular}

Values are presented as mean \pm standard deviation or number (\%).

FRAIL scale, Fatigue, Resistance, Ambulation, Illnesses, \& Loss of Weight scale; BMI, body mass index; SPPB, Short Physical Performance Battery; ADL, activity of daily living; IADL, instrumental activity of daily living.

${ }^{\text {a) }}$ Kruskal-Wallis test.

Table 2. Associations between frailty status and geriatric conditions

\begin{tabular}{|c|c|c|c|c|c|}
\hline Outcome & Level & Cases & $\mathrm{OR}^{\mathrm{a})}$ & $95 \% \mathrm{CI}$ & p-value \\
\hline \multirow[t]{3}{*}{ Living alone $(\mathrm{n}=341)$} & Robust (Ref) & 91 & - & - & \\
\hline & Prefrail & 149 & 1.13 & $0.82-1.56$ & 0.462 \\
\hline & Frail & 101 & 1.08 & $0.73-1.61$ & 0.702 \\
\hline \multirow[t]{3}{*}{ Low income $(\mathrm{n}=88)$} & Robust (Ref) & 21 & - & - & \\
\hline & Prefrail & 37 & 1.43 & $0.81-2.52$ & 0.220 \\
\hline & Frail & 30 & 1.61 & $0.83-3.13$ & 0.158 \\
\hline \multirow[t]{3}{*}{ Multimorbidity $(\mathrm{n}=517)$} & Robust (Ref) & 139 & - & - & \\
\hline & Prefrail & 210 & 1.33 & $1.01-1.75$ & 0.047 \\
\hline & Frail & 168 & 2.24 & $1.57-3.21$ & $<0.001$ \\
\hline \multirow[t]{3}{*}{ Dysmobility $(n=363)$} & Robust (Ref) & 61 & - & - & \\
\hline & Prefrail & 133 & 1.53 & $1.07-2.19$ & 0.02 \\
\hline & Frail & 169 & 5.35 & $3.58-7.97$ & $<0.001$ \\
\hline \multirow[t]{3}{*}{ Malnutrition $(n=64)$} & Robust (Ref) & 1 & - & - & \\
\hline & Prefrail & 20 & 2.29 & $1.72-3.06$ & $<0.001$ \\
\hline & Frail & 43 & 4.09 & $2.83-5.90$ & $<0.001$ \\
\hline \multirow[t]{3}{*}{ Impaired cognition $(n=383)$} & Robust (Ref) & 62 & - & - & \\
\hline & Prefrail & 164 & 1.96 & $1.38-2.79$ & $<0.001$ \\
\hline & Frail & 157 & 3.52 & $2.36-5.25$ & $<0.001$ \\
\hline \multirow[t]{3}{*}{ Depressive mood $(\mathrm{n}=142)$} & Robust (Ref) & 6 & - & - & \\
\hline & Prefrail & 49 & 6.24 & $2.62-14.9$ & $<0.001$ \\
\hline & Frail & 87 & 27.50 & $11.09-68.19$ & $<0.001$ \\
\hline \multirow[t]{3}{*}{ Disability $(\mathrm{n}=615)$} & Robust (Ref) & 128 & - & - & \\
\hline & Prefrail & 225 & 1.12 & $0.84-1.50$ & 0.448 \\
\hline & Frail & 262 & 3.12 & $2.18-4.46$ & $<0.001$ \\
\hline
\end{tabular}

OR, odds ratio; CI, confidence interval.

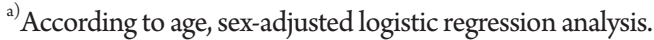


deterioration of the quality of life (Table 3). The differences in 3-year mortality according to the FRAIL scale are shown in Fig. 1. The frail group had significantly lower estimated median survival (203 months) than the prefrail (410 months) and robust (357 months) groups at 30 months follow-up (all p $<0.05$ ).

\section{DISCUSSION}

The results of this prospective study showed that frailty according to the K-FRAIL scale was associated with accompanying geriatric conditions and with 3-year health-related outcomes such as mortality, institutionalization, and disability in community-dwelling Korean older adults.

The K-FRAIL scale showed high sensitivity and negative predictive value in differentiating vulnerability from robustness; however, the quantification of frailty status with respect to various health-related outcomes has been understudied. The clinical implications of our findings are that frail individuals identified using the K-FRAIL scale have an elevated disability, institutionalization, and mortality profile. Therefore, these frail individuals might benefit from further comprehensive geriatric assessments and multidomain interventions. The K-FRAIL scale is correlated with the previously validated frailty index and other frailty measures. ${ }^{16)}$ Our results provide evidence for the adequacy of the FRAIL scale, including its associations with health-related outcomes, as well as identifying individuals with a high burden of geriatric conditions in rural communities.

A previous systematic review and meta-analysis of 3-5 studies demonstrated high $\mathrm{ADL}$ (pooled $\mathrm{OR}=9.82$, compared with robustness) and IADL (pooled $\mathrm{OR}=2.50$, compared with robustness) disability risks for frailty according to the FRAIL scale. ${ }^{20)}$

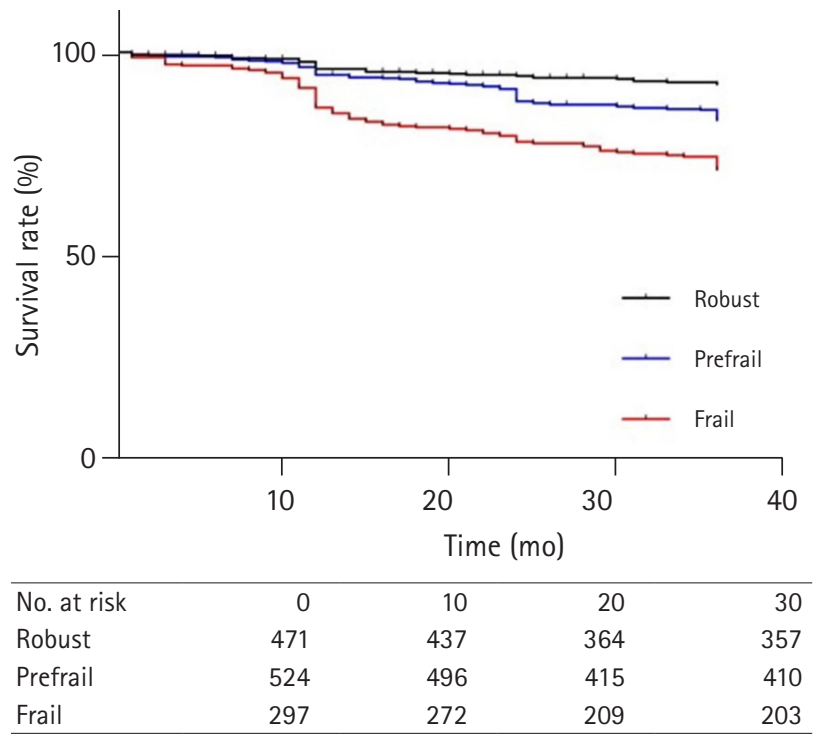

Fig. 1. Differences in 3-year mortality according to the FRAIL (Fatigue, Resistance, Ambulation, Illnesses, \& Loss of Weight) scale.

Table 3. Association between FRAIL scale and health outcomes

\begin{tabular}{|c|c|c|c|c|}
\hline Outcome & Level & $\mathrm{OR}^{\mathrm{a})}$ & $95 \%$ CI & $\mathrm{p}$-value \\
\hline \multirow[t]{3}{*}{ Mortality } & Robust (Ref) & - & - & \\
\hline & Prefrail & 1.53 & $0.78-2.98$ & 0.214 \\
\hline & Frail & 3.57 & $1.69-7.54$ & $<0.001$ \\
\hline \multirow[t]{3}{*}{ Institution } & Robust (Ref) & - & - & \\
\hline & Prefrail & 1.69 & $0.96-2.95$ & 0.068 \\
\hline & Frail & 2.42 & $1.30-4.51$ & 0.005 \\
\hline \multirow[t]{3}{*}{ Incidence of disability } & Robust (Ref) & - & - & \\
\hline & Prefrail & 1.58 & $1.10-2.27$ & 0.013 \\
\hline & Frail & 3.04 & $1.91-4.85$ & $<0.001$ \\
\hline \multirow[t]{3}{*}{ Mortality or institution } & Robust (Ref) & - & - & \\
\hline & Prefrail & 1.70 & $1.09-2.67$ & 0.02 \\
\hline & Frail & 3.14 & $1.90-5.18$ & $<0.001$ \\
\hline \multirow[t]{3}{*}{ Mortality, institution, or incident disability } & Robust (Ref) & - & - & \\
\hline & Prefrail & 1.67 & $1.22-2.29$ & 0.001 \\
\hline & Frail & 3.78 & $2.52-5.66$ & $<0.001$ \\
\hline \multirow[t]{3}{*}{ EQ-5D decrease of $\geq 0.2$} & Robust (Ref) & - & - & \\
\hline & Prefrail & 1.44 & $0.67-3.09$ & 0.350 \\
\hline & Frail & 0.57 & $0.19-1.69$ & 0.315 \\
\hline
\end{tabular}

FRAIL scale, Fatigue, Resistance, Ambulation, Illnesses, \& Loss of Weight scale; OR, odds ratio; CI, confidence interval; EQ-5D, EuroQol-5D.

${ }^{\text {a) }}$ For 3-year outcomes according to age, gender-adjusted logistic regression analysis. 
Our results using the K-FRAIL scale were consistent with previous results. Similar to our results, another meta-analysis of three studies showed that both frailty (pooled $\mathrm{OR}=3.53$, compared with robustness) and prefrailty (pooled $\mathrm{OR}=1.75$ compared with robustness) were significantly associated with higher mortality risk. ${ }^{18)} \mathrm{Al}$ though previous studies less often focused on institutionalization as an outcome of the FRAIL scale, we found that frailty according to the K-FRAIL scale was associated with an increased risk of longterm institutionalization. The results of our cross-sectional analysis showed that frailty was highly associated with depressed mood, dysmobility, risk of malnutrition, and multimorbidity. The fact that the FRAIL scale encompasses comorbidities should be considered when interpreting multimorbidity rates.

However, the longitudinal analysis showed that baseline frailty status was not associated with future QOL. Although the mechanism is not clear in the current study, the lack of association between the FRAIL scale and future QOL decline might be due to the characteristics of the FRAIL scale rather than the clinical construct of frailty itself, with a previous study being able to produce meaningful changes in frailty status from the same study population using EQ-5D as an anchor. ${ }^{30)}$

Our study has several strengths and limitations. The limitation was that the results were obtained from underserved older populations in rural areas, which restricted their generalizability to the general population of Korea. However, this cohort study included more than $90 \%$ of eligible older adults, with high completion rates of geriatric parameters in the study population. The outcomes of functional changes were available in our study population because the study population remained relatively stable, with $<5 \%$ of older adults immigrating or emigrating from the area except for morbidity or mortality, with the study area rather distant from major cities in Korea. Residents received medical and preventive care almost exclusively from regional community health posts operated by the National Health Service. Therefore, we could examine long-term clinical outcomes such as mortality and institutionalization.

In conclusion, frailty according to the five-item FRAIL scale was associated with geriatric syndromes and 3-year health-related outcomes in rural community-dwelling Korean older adults. In the context of public health, this simple screening tool for frailty assessment might be applicable to older populations in Korea.

\section{ACKNOWLEDGEMENTS}

We are indebted to the public health professionals and nurses of Pyeonchang County Hospital, Public Health Center, and Community Health Posts for their administrative support and efforts in participant enrollment, retention, and measurements.

\section{CONFLICT OF INTEREST}

The researchers claim no conflicts of interest.

\section{FUNDING}

This study was supported by the Korean Geriatrics Society Research Challenge Award (2019).

\section{AUTHOR CONTRIBUTION}

Conceptualization, HL, JYC, HWJ, IYJ; data acquisition, IYJ; data analysis, HL, JYC, IYJ; writing, original draft, HL, JYC, HWJ, IYJ; writing review and editing, HL, JYC, HWJ, JYB, EL, IYJ.

\section{REFERENCES}

1. Kontis V, Bennett JE, Mathers CD, Li G, Foreman K, Ezzati M. Future life expectancy in 35 industrialised countries: projections with a Bayesian model ensemble. Lancet 2017;389:1323-35.

2. Jang IY, Lee HY; Lee E; 50th Anniversary Committee of Korean Geriatrics Society. Geriatrics Fact Sheet in Korea 2018 from national statistics. Ann Geriatr Med Res 2019;23:50-3.

3. Fried LP, Tangen CM, Walston J, Newman AB, Hirsch C, Gottdiener J, et al. Frailty in older adults: evidence for a phenotype. J Gerontol A Biol Sci Med Sci 2001;56:M146-56.

4. Kojima T. The need for actions against polypharmacy in older people with frailty. Ann Geriatr Med Res 2018;22:111-6.

5. Kim MY, Etherton-Beer C, Kim CB, Yoon JL, Ga H, Kim HC, et al. Development of a consensus list of potentially inappropriate medications for Korean older adults. Ann Geriatr Med Res 2018;22:121-9.

6. Jang IY, Lee E, Lee H, Park H, Kim S, Kim KI, et al. Characteristics of sarcopenia by European consensuses and a phenotype score. J Cachexia Sarcopenia Muscle 2020;11:497-504.

7. Ga H, Won CW, Jung HW. Use of the frailty index and FRAIL$\mathrm{NH}$ scale for the assessment of the frailty status of elderly individuals admitted in a long-term care hospital in Korea. Ann Geriatr Med Res 2018;22:20-5.

8. Kojima G. Frailty as a predictor of future falls among community-dwelling older people: a systematic review and meta-analysis. J Am Med Dir Assoc 2015;16:1027-33.

9. Ensrud KE, Ewing SK, Cawthon PM, Fink HA, Taylor BC, Cauley JA, et al. A comparison of frailty indexes for the prediction of falls, disability, fractures, and mortality in older men. J Am Geriatr Soc 2009;57:492-8.

10. Jung HW, Kang MG, Choi JY, Yoon SJ, Kim SW, Kim KI, et al. Simple method of screening for frailty in older adults using a chronometer and tape measure in clinic. J Am Geriatr Soc 2018;66:157-60. 
11. Rockwood K, Song X, MacKnight C, Bergman H, Hogan DB, $\mathrm{McDowell} I$, et al. A global clinical measure of fitness and frailty in elderly people. CMAJ 2005; 173:489-95.

12. Lee H, Lee E, Jang IY. Frailty and comprehensive geriatric assessment. J Korean Med Sci 2020;35:e16.

13. Morley JE, Malmstrom TK, Miller DK. A simple frailty questionnaire (FRAIL) predicts outcomes in middle aged African Americans. J Nutr Health Aging 2012;16:601-8.

14. Malmstrom TK, Miller DK, Morley JE. A comparison of four frailty models. J Am Geriatr Soc 2014;62:721-6.

15. Susanto M, Hubbard RE, Gardiner PA. Validity and responsiveness of the FRAIL scale in middle-aged women. J Am Med Dir Assoc 2018; 19:65-9.

16. Jung HW, Yoo HJ, Park SY, Kim SW, Choi JY, Yoon SJ, et al. The Korean version of the FRAIL scale: clinical feasibility and validity of assessing the frailty status of Korean elderly. Korean J Intern Med 2016;31:594-600.

17. Jang IY, Jung HW, Lee CK, Jang KH, Cho EI, Jung JJ, et al. Korean version of the Fatigue, Resistance, Ambulation, Illnesses and Loss of weight questionnaire versus the Modified Kihon Checklist for frailty screening in community-dwelling older adults: the aging study of PyeongChang rural area. Geriatr Gerontol Int 2017;17:2046-52.

18. Kojima G. Frailty defined by FRAIL scale as a predictor of mortality: a systematic review and meta-analysis. J Am Med Dir Assoc 2018; 19:480-3.

19. Thompson MQ, Theou O, Tucker GR, Adams RJ, Visvanathan R. FRAIL scale: predictive validity and diagnostic test accuracy. Australas J Ageing 2020;39:e529-e536.

20. Kojima G. Quick and simple FRAIL scale predicts incident activities of daily living (ADL) and instrumental ADL (IADL) disabilities: a systematic review and meta-analysis. J Am Med Dir Assoc 2018;19:1063-8.

21. Won CW, Yang KY, Rho YG, Kim SY, Lee EJ, Yoon JL, et al. The development of Korean activities of daily living (K-ADL) and Korean instrumental activities of daily living (K-IADL) scale. J Korean Geriatr Soc 2002;6:107-20.

22. American Geriatrics Society 2012 Beers Criteria Update Expert Panel. American Geriatrics Society updated Beers Criteria for potentially inappropriate medication use in older adults. J Am Geriatr Soc 2012;60:616-31.

23. Kang Y, Na DL, Hahn S. A validity study on the Korean Mini-Mental State Examination (K-MMSE) in dementia patients. J Korean Neurol Assoc 1997;15:300-8.

24. Radloff LS. The CES-D scale: a self-report depression scale for research in the general population. Appl Psychol Meas 1977; $1: 385-401$.

25. Rubenstein LZ, Harker JO, Salva A, Guigoz Y, Vellas B. Screening for undernutrition in geriatric practice: developing the shortform mini-nutritional assessment (MNA-SF). J Gerontol A Biol Sci Med Sci 2001;56:M366-72.

26. Cummings SR, Studenski S, Ferrucci L. A diagnosis of dismobility: giving mobility clinical visibility: a Mobility Working Group recommendation.JAMA 2014;311:2061-2.

27. Guralnik JM, Simonsick EM, Ferrucci L, Glynn RJ, Berkman LF, Blazer DG, et al. A short physical performance battery assessing lower extremity function: association with self-reported disability and prediction of mortality and nursing home admission. J Gerontol 1994;49:M85-94.

28. Chen LK, Liu LK, Woo J, Assantachai P, Auyeung TW, Bahyah KS, et al. Sarcopenia in Asia: consensus report of the Asian Working Group for Sarcopenia. J Am Med Dir Assoc 2014;15: 95-101.

29. EuroQol Group. EuroQol: a new facility for the measurement of health-related quality of life. Health Policy 1990;16:199-208.

30. Jang IY, Jung HW, Lee HY, Park H, Lee E, Kim DH. Evaluation of clinically meaningful changes in measures of frailty. J Gerontol A Biol Sci Med Sci 2020;75:1143-7. 